\title{
Scalar Non Standard Interactions at long baseline experiments
}

\author{
Abinash Medhi, ${ }^{a, *}$ Debajyoti Dutta ${ }^{b}$ and Moon Moon Devi ${ }^{a}$ \\ ${ }^{a}$ Department of Physics, Tezpur University, \\ Napaam, Sonitpur, Assam-784028, India \\ ${ }^{b}$ Department of Physics, Assam Don Bosco University, \\ Kamarkuchi, Sonapur, Assam-782402, India \\ E-mail: amedhi@tezu.ernet.in, debajyoti.dutta@dbuniversity.ac.in, \\ devimm@tezu.ernet.in
}

The discovery of neutrino oscillations confirms neutrinos have mass and the Standard Model(SM) of particle physics is not complete. It needs revision in order to accommodate the masses and mixing of neutrinos, which essentially leads to beyond SM (BSM) physics. The unknown couplings involving neutrinos, so-called the Non-Standard Interactions(NSIs) [1, 3] may appear as a 'new physics' in different neutrino experiments. Neutrino NSI can have a sizable impact on neutrino oscillation and can impact the measurements of different mixing parameters in various neutrino experiments. The recent work on scalar NSI [4, 5], has shown a great potential to probe it further. Unlike vector NSI, scalar NSI appears as a correction to the neutrino mass matrix rather than acting as a matter potential. This may lead to a significantly different phenomenological consequence in different neutrino experiments. Moreover, as scalar NSI affects the mass matrix, it also gives a possibility of probing it further to different neutrino mass models.

In this work, we explored the effect of scalar NSI at long-baseline experiment DUNE [6]. We point out that scalar NSI can considerably affect the neutrino oscillation in Long baseline(LBL) experiments and can complicate the measurement of the $\mathrm{CP}$ phase. Also as it appears as a correction to the neutrino mass matrix its effect is energy independent, unlike the vector NSI. We also studied the sensitivity of different LBL experiments towards finding the effects of scalar NSI. Also, we put up the possibility of probing it further to various neutrino mass models.

$37^{\text {th }}$ International Cosmic Ray Conference (ICRC 2021)

July 12th - 23rd, 2021

Online - Berlin, Germany

\footnotetext{
*Presenter
} 


\section{Introduction}

The discovery of neutrino oscillation confirms that neutrinos have masses and the neutrino flavours mixes with one another. The phenomena of neutrino oscillation has played an important role in our understanding of various properties of neutrinos. The SM of particle physics does not give masses to neutrinos and it needs to be extended in order to explain the masses of neutrinos. These kind of extension in general comes with some additional unknown couplings of neutrinos. These coupling are beyond the scope of SM, so these interactions are generally termed as Non Standard Interactions (NSIs). The subdominant effects due to NSIs can significantly effect the measurement of various mixing parameters in different neutrino experiments.

The idea of NSI was first introduced by Wolfenstein in in landmark paper [2] of neutrino matter interactions. In that paper he mentioned that even the massless neutrinos can oscillate from one flavour to another due to NSI. Later the idea of NSI has been widely explored by different authors and it is found that indeed understanding the effects of NSI can be crucial in our understanding of neutrino parameters.

The coupling of neutrinos with a scalar is quite promising and exciting [4]. Unlike the conventional vector type NSI where the interactions is mediated by a vector mediator, scalar type NSI effects differently in the neutrino propagation. The scalar NSI appears as a correction to the mass matrix in the neutrino Hamiltonian. Also, the effects of scalar NSI is independent of neutrino energy, hence it is not suppressed at low energy. As, scalar NSI directly depends on the matter density, Long Baseline (LBL) neutrino experiments are excellent candidate to probe it in neutrino oscillations.

\section{Formalism}

Within the framework of SM neutrinos interact with matter very weakly via charged current (CC) or neutral current (NC) interactions. The mediator of CC interaction and NC interactions are $\mathrm{W}$ and $\mathrm{Z}$ boson respectively. Following the theory of SM interactions these interactions can be written in the form $\left(\bar{v}_{\alpha} \gamma^{\mu} P_{L} v_{\alpha}\right)\left(\bar{f} \gamma_{\mu} P f\right)$ (with $f, f^{\prime} \in\{e, u, d\}$ the matter fermions and $P \in\left\{P_{L}, P_{R}\right\}$ the chirality projection operators. The chiral projection operators are given by $P_{L}=\left(1-\gamma_{5}\right) / 2$ and $\left.P_{R}=\left(1+\gamma_{5}\right) / 2\right)$. The effective Lagrangian for neutrino matter interactions can be formed as :

$$
\mathcal{L}_{\mathrm{cc}}^{\mathrm{eff}}=-\frac{4 G_{F}}{\sqrt{2}}\left[\overline{v_{e}}\left(p_{3}\right) \gamma_{\mu} P_{L} v_{e}\left(p_{2}\right)\right]\left[\bar{e}\left(p_{1}\right) \gamma^{\mu} P_{L} e\left(p_{4}\right)\right]
$$

Where, $G_{F}$ is the Fermi constant. The neutrino matter effects comes due to the forward scattering of neutrinos and here only the CC current interaction will only effect the neutrino oscillations. The effective matter potential due to CC interactions can be written as $V_{\mathrm{cc}}=-\bar{V}_{\mathrm{cc}}=\sqrt{2} G_{F} n_{e}$. where, $V_{\mathrm{cc}}$ is for neutrino case and $\bar{V}_{\mathrm{cc}}$ is for antineutrino case and $n_{e}$ is the number density of environmental electrons. The effective Hamiltonian in presence of neutrino matter effect can be written as : 


$$
\mathcal{H} \approx E_{v}+\frac{M M^{\dagger}}{2 E_{v}} \pm V_{\mathrm{SI}}
$$

Where $E_{v}$ represents the neutrino energy, $\mathrm{M}$ is the neutrino mass matrix and $V_{S I}$ is the matter potential due to forward scattering of neutrinos with matter. The ' + ' and the '-' sign before the neutrino standard interaction potential $\left(V_{S I}\right)$ corresponds to neutrino and antineutrino mode respectively.

So, the neutrino matter effect appears as an extra matter potential in the neutrino Hamiltonian. The conventional vector type NSI which comes due to introducing a vector currents from a vector mediator or with Fierz transformation from a charged scalar also appears as a matter potential in the neutrino Hamiltonian. Hence its effect is also matter potential. In addition there is also possibility of neutrinos coupling with a scalar. These kind of interactions are generally termed as scalar NSI. The Lagrangian for such scalar NSI can be framed as:

$$
\mathcal{L}_{\mathrm{eff}}^{\mathrm{S}}=\frac{y_{f} y_{\alpha \beta}}{m_{\phi}^{2}}\left(\bar{v}_{\alpha}\left(p_{3}\right) v_{\beta}\left(p_{2}\right)\right)\left(\bar{f}\left(p_{1}\right) f\left(p_{4}\right)\right)
$$

Where, $\alpha, \beta=\mathrm{e}, \mu, \tau$ refer to the neutrino flavours, $\mathrm{f}=\mathrm{e}, \mathrm{u}, \mathrm{d}$ indicate the matter fermions, $y_{\alpha \beta}$ is the Yukawa couplings of the neutrinos with the scalar mediator, $\phi$ and $y_{f}$ is the Yukawa coupling of the scalar mediator $\phi$ with the environmental fermions $f$. The $m_{\phi}$ is the mass of the scalar mediator $\phi$. So, the effective Hamiltonian in presence of scalar NSI modifies as :

$$
\mathcal{H} \approx E_{v}+\frac{(M+\delta M)(M+\delta M)^{\dagger}}{2 E_{v}} \pm V_{\mathrm{SI}}
$$

Where, the expression for $\delta M$ is defined as $\delta M \equiv \sum_{f} n_{f} y_{f} y_{\alpha \beta} / m_{\phi}^{2}$. Here $n_{f}$ is the number density of environmental fermion. So, the effect of scalar NSI appears as a correction or addition to the neutrino mass term. The mass correction matrix can be parametrized as :

$$
\delta M \equiv \sqrt{\Delta m_{31}^{2}}\left(\begin{array}{lll}
\eta_{e e} & \eta_{e \mu} & \eta_{e \tau} \\
\eta_{\mu e} & \eta_{\mu \mu} & \eta_{\mu \tau} \\
\eta_{\tau e} & \eta_{\tau \mu} & \eta_{\tau \tau}
\end{array}\right)
$$

The $\eta_{\alpha \beta}$ parameters are complex and it quantifies the size of scalar NSI. Also, the hermicity of the neutrino Hamiltonian requires the diagonal terms to be real and off diagonal terms to be complex. These parameters are dimensionless and can be parametrized as: $\eta_{\alpha \beta}=\left|\eta_{\alpha \beta}\right| e^{i \phi_{\alpha \beta}}$; for $\alpha \neq \beta$. 


\section{Effects on oscillation probability}

In our work, we have we have checked the effects of scalar NSI on the oscillation probability of DUNE. We have particularly checked the effects on two probability channel i. e. appearance probability $\left(v_{\mu} \rightarrow v_{e}\right)$ and disappearance probability $\left(v_{\mu} \rightarrow v_{\mu}\right)$.

The details of the DUNE experiment used in our analysis are listed in Table 1. The details of the true values of mixing parameters used in our analysis are listed in Table 2.

\begin{tabular}{|c|c|c|c|c|c|}
\hline Experiment & Signal & $\begin{array}{c}\text { Signal } \\
\text { Efficiencies }\end{array}$ & $\begin{array}{c}\text { Energy } \\
\text { Resolutions }\end{array}$ & $\begin{array}{c}\text { Runtime (yrs) } \\
v+\bar{v}\end{array}$ & Detector Mass (Type) \\
\hline DUNE & $v_{e}^{C C}$ & $80 \%$ & $0.15 / \sqrt{E}$ & $5+5$ & 35 kton (LArTPC) \\
& $v_{\mu}^{C C}$ & $85 \%$ & $0.20 / \sqrt{E}$ & & \\
\hline
\end{tabular}

Table 1: Simulation details like signal efficiencies, energy resolutions, total exposures and detector mass of DUNE

\begin{tabular}{|c|c|}
\hline Parameters & True Values \\
\hline$\theta_{12}$ & $34.51^{\circ}$ \\
$\theta_{13}$ & $8.44^{\circ}$ \\
$\theta_{23}$ & $47^{\circ}$ \\
$\delta_{C P}$ & $-\pi / 2$ \\
$\Delta m_{21}^{2}$ & $7.56 \times 10^{-5}$ \\
$\Delta m_{31}^{2}$ & $2.43 \times 10^{-3}$ \\
\hline
\end{tabular}

Table 2: Oscillation parameters used in probability analysis

Figure 1 and Figure 2 shows the effects of diagonal scalar NSI elements $\eta_{e e}$ and $\eta_{\mu \mu}$ on the oscillation probabilities of DUNE. We have varied the energy from 0.5 to $10 \mathrm{GeV}$. The red line represents the standard interaction (SI) case with zero $\eta$ values. The black and blue lines are cases with different values of $\eta$. In all the plots $\mathrm{NH}$ is considered as the true mass hierarchy. It can be seen that the effects of scalar NSI on the oscillation probability is significant. $\eta_{e e}$ suppresses the probability at the oscillation peaks whereas $\eta_{\mu \mu}$ enhances the probability at the peaks. The probability plots for positive and negative non zero $\eta$ elements are symmetric around zero.

Figure 3 and 4 represents how appearance probability $\left(P_{\mu e}\right)$ is varied with different $\delta_{C P}$ for DUNE at a fixed energy of $2.5 \mathrm{GeV}$. Here also the solid red line represents the SI case. From the plot it can be seen that there is a strong correlation between NSI parameters with the $\delta_{C P}$ and the probabilities. It also shows the presence of various degeneracy for different values of $\eta$. Here also the probability plots for positive and negative non zero $\eta$ elements are symmetric around zero.

\section{Results and Discussions}

\subsection{Details of $\chi^{2}$ analysis}

We have done a sensitivity analysis to check whether DUNE can distinguish the scalar NSI effects from SI case. We have used the following definition of $\chi^{2}$ in our analysis. 


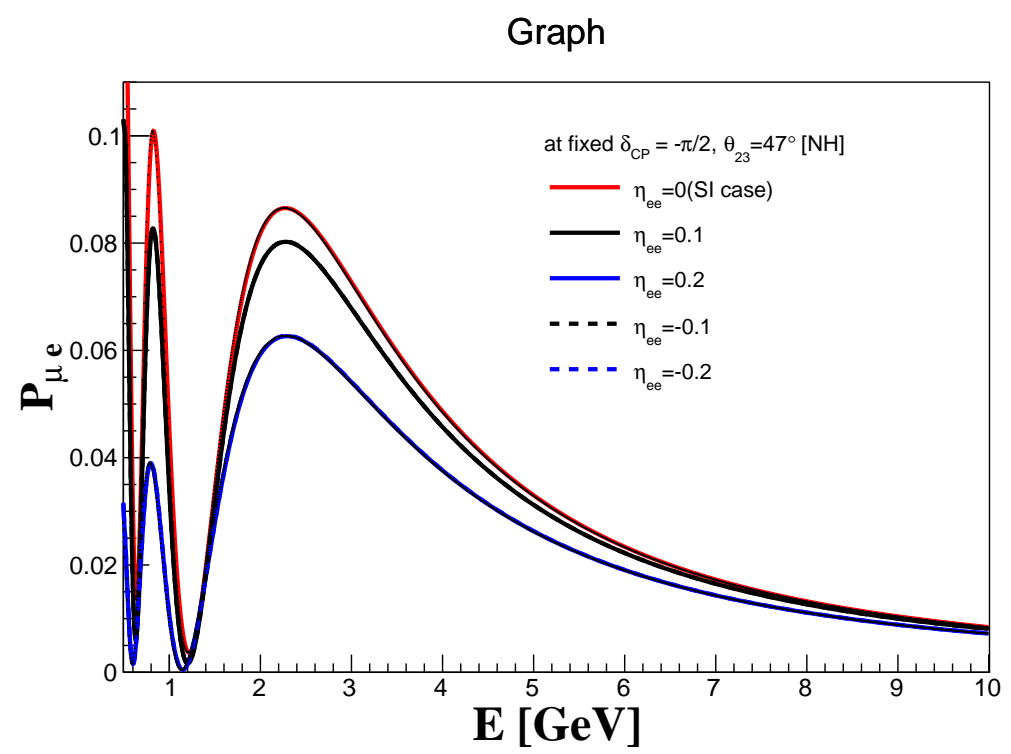

Figure 1: The effect of the diagonal scalar NSI elements, $\eta_{e e}$ and on neutrino appearance probabilities $\left(P_{\mu e}\right)$ at DUNE (baseline $=1300 \mathrm{~km}$ ).

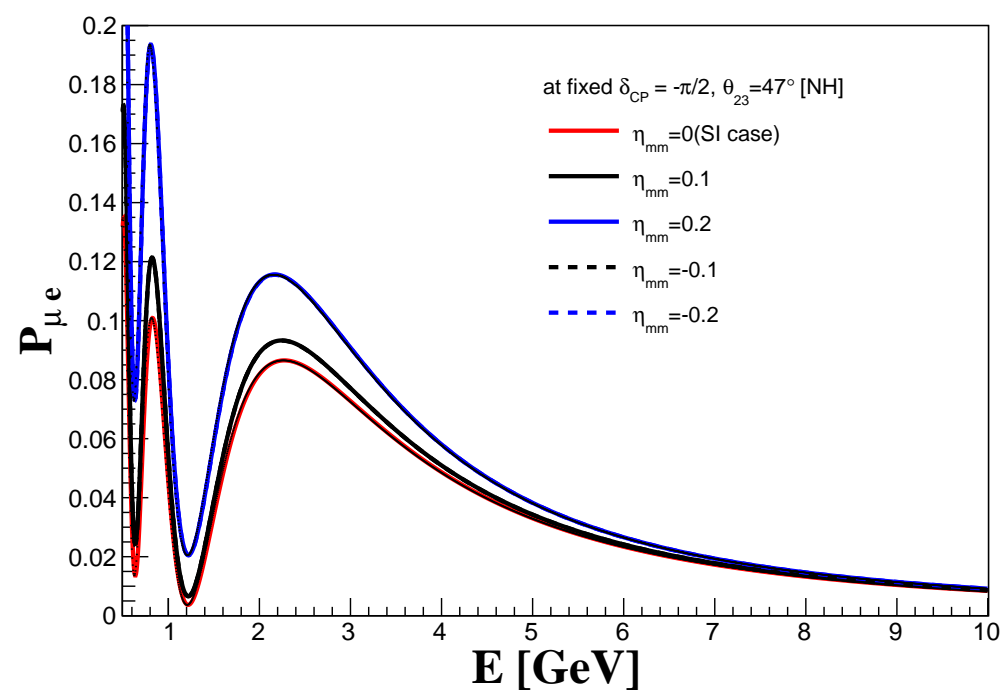

Figure 2: The effect of the diagonal scalar NSI elements $\eta_{\mu \mu}$ on neutrino appearance probabilities $\left(P_{\mu e}\right)$ at DUNE (baseline $=1300 \mathrm{~km}$ ).

$$
\chi^{2} \equiv \min _{\eta} \sum_{i} \sum_{j} \frac{\left[N_{\text {true }}^{i, j}(\eta)-N_{\text {test }}^{i, j}(\eta)\right]^{2}}{N_{\text {true }}^{i, j}(\eta)}
$$

where $N_{\text {true }}^{i, j}$ and $N_{\text {test }}^{i, j}$ are the number of true and test events in the $\{i, j\}$-th bin respectively. We have made a framework with GLoBES [7] to perform our simulation studies. For our $\chi^{2}$ 


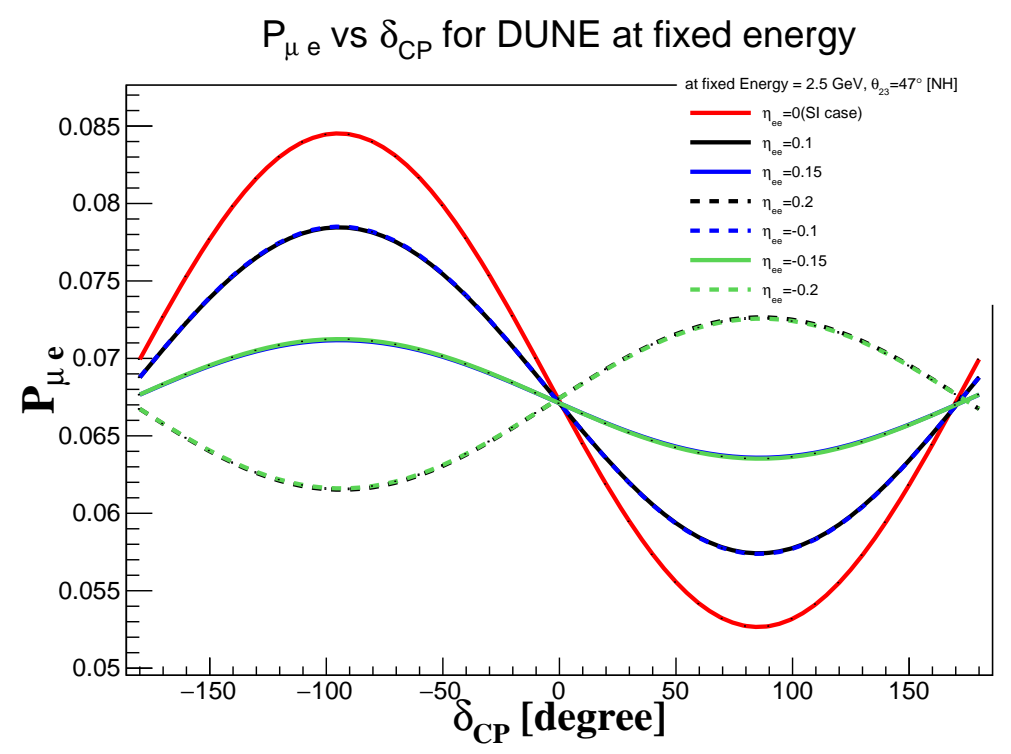

Figure 3: The effect of the diagonal scalar NSI elements $\eta_{\mu \mu}$ on neutrino appearance probabilities $\left(P_{\mu e}\right)$ at DUNE (baseline $=1300 \mathrm{~km}$ ) for fixed $\delta_{C P}=-\pi / 2, \theta_{23}=47^{\circ}$ and energy $=2.5 \mathrm{GeV}$.

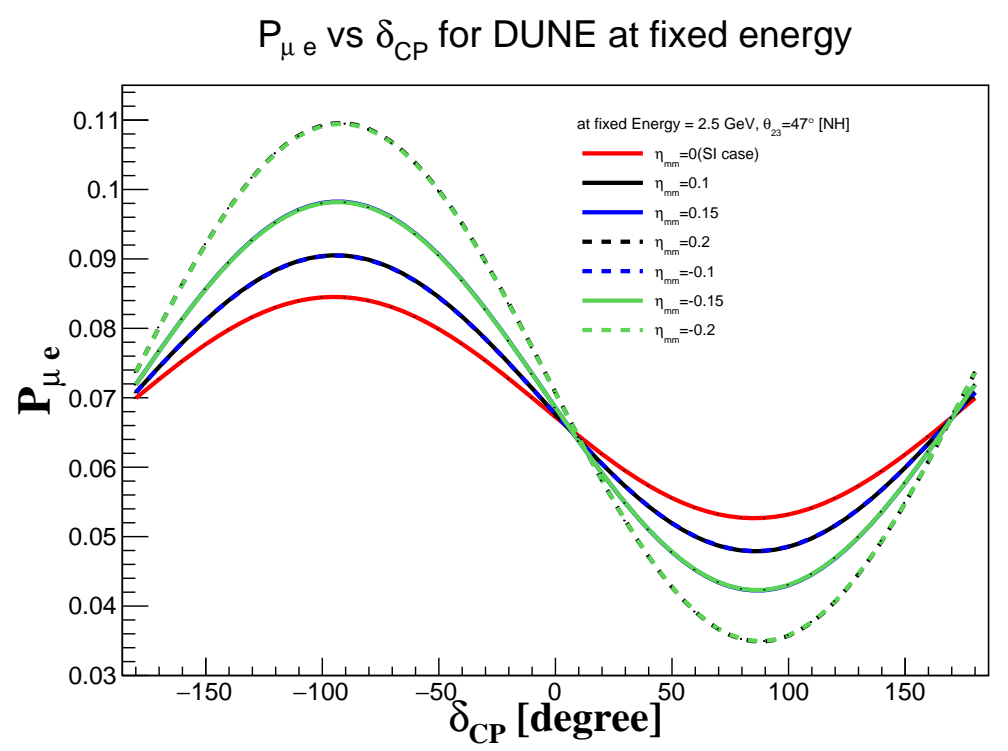

Figure 4: The effect of the diagonal scalar NSI elements $\eta_{\mu \mu}$ on neutrino appearance probabilities $\left(P_{\mu e}\right)$ at DUNE (baseline $=1300 \mathrm{~km}$ ) for fixed $\delta_{C P}=-\pi / 2, \theta_{23}=47^{\circ}$ and energy $=2.5 \mathrm{GeV}$.

study, we have fixed all the true values of the mixing parameters and also the values of scalar NSI parameters. All the true values of diagonal scalar NSI elements $\left(\eta_{e e}, \eta_{\mu \mu}\right.$ and $\left.\eta_{\tau \tau}\right)$ are set at a fixed value of 0.01 and all the remaining off-diagonal $\eta$ parameters and phases are taken as zeros. Finally, we have marginalized all the test values of diagonal $\eta$ elements in the range -0.15 to 0.15 for each plot of the fixed $\chi^{2}$ analysis. 


\subsection{Analysis Results}

Figure 5 represents the sensitivity of DUNE towards finding the diagonal scalar NSI parameters. The red solid line corresponds to true $\eta_{e e}=0.01$ and the black and blue dotted line correspond to true $\eta_{\mu \mu}=0.01$ and true $\eta_{\tau \tau}=0.01$ respectively. The plot for $\eta_{\mu \mu}$ and $\eta_{\tau \tau}$ is exactly same, only the effect of $\eta_{e e}$ it is different. The sensitivity of finding the $\eta_{e e}$ elements is little less for DUNE is as compared to the $\eta_{\mu \mu}$ and $\eta_{\tau \tau}$ elements.

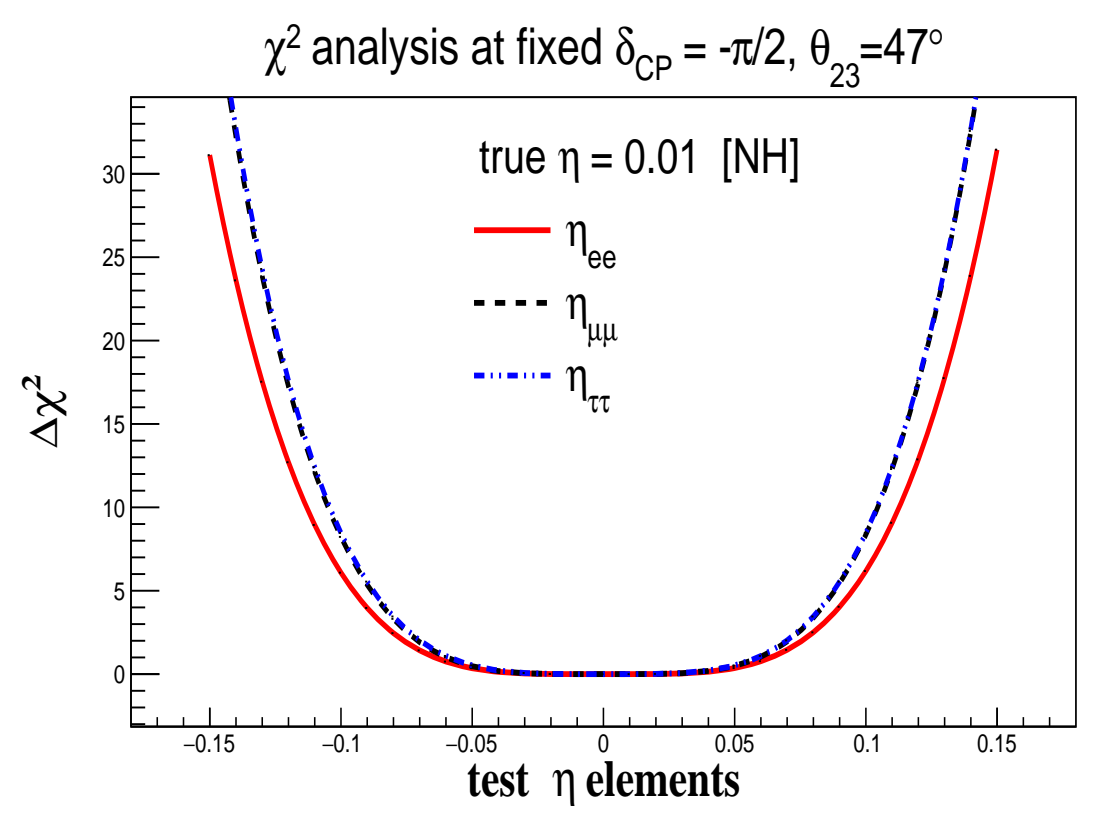

Figure 5: Fixed $\chi^{2}$ analysis for DUNE in presence of diagonal scalar NSI elements for true $\delta_{C P}=-\pi / 2$ and true $\theta_{23}=47^{\circ}$.

Figure 6 shows the CP sensitivity of DUNE in presence of diagonal scalar NSI elements $\eta_{e e}$. The red line represents the SI case without scalar NSI. We have seen that with increase in the values of scalar NSI parameter the CP sensitivity of DUNE gets deteriorated. For the values of $\eta_{e e}=0.15$ and higher the CP sensitivity of DUNE lies below $3 \sigma$.

\section{Summary and Concluding Remarks}

To summarise, we found that the impact of scalar NSI on oscillation probabilities at DUNE is significant. It is an excellent candidate to probe new physics Beyond SM . Also, the CP sensitivity of DUNE gets spoiled due to inclusion of scalar NSI. Moreover, It is important to identify these subdominant effects due to scalar NSI for accurate interpretation of data from various neutrino experiments. Finally as it effects the mass term, probing it to various neutrino mass models in quite interesting and promising.

\section{Acknowledgement}

The work is supported by the Research and Innovation grant 2021 (DoRD/RIG/10-73/ 1592A), Tezpur University. MMD would like to acknowledge the support of DST SERB grant 


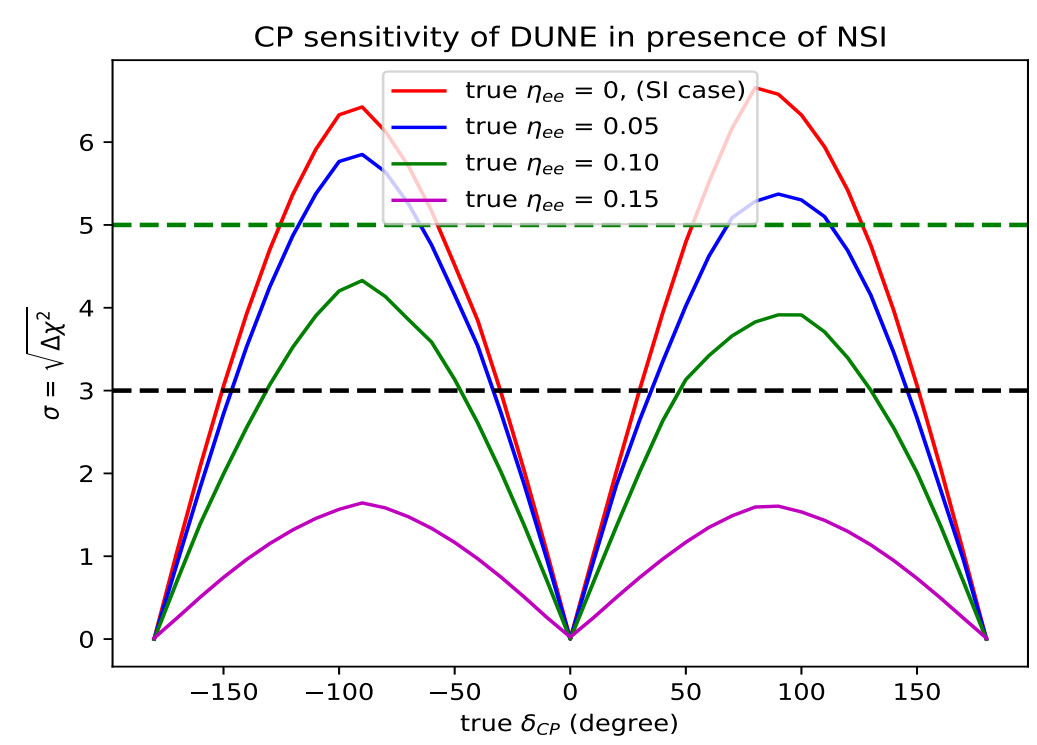

Figure 6: The plot represents CP sensitivity of DUNE (baseline $=1300 \mathrm{~km}$ ) in presence of scalar NSI .

EMR/2017/001436 for this work.

\section{References}

[1] O. G. Miranda and H. Nunokawa, New J. Phys. 17, no.9, 095002 (2015) doi:10.1088/13672630/17/9/095002 [arXiv:1505.06254 [hep-ph]].

[2] L. Wolfenstein, Phys. Rev. D 17 (1978), 2369-2374 doi:10.1103/PhysRevD.17.2369

[3] Y. Farzan and M. Tortola, Front. in Phys. 6 (2018), 10 doi:10.3389/fphy.2018.00010 [arXiv:1710.09360 [hep-ph]].

[4] S. F. Ge and S. J. Parke, Phys. Rev. Lett. 122 (2019) no.21, 211801 doi:10.1103/PhysRevLett.122.211801 [arXiv:1812.08376 [hep-ph]].

[5] K. S. Babu, G. Chauhan and P. S. Bhupal Dev, Phys. Rev. D 101 (2020) no.9, 095029 doi:10.1103/PhysRevD.101.095029 [arXiv:1912.13488 [hep-ph]].

[6] B. Abi et al. [DUNE], JINST 15 (2020) no.08, T08010 doi:10.1088/1748-0221/15/08/T08010 [arXiv:2002.03010 [physics.ins-det]].

[7] P. Huber, M. Lindner and W. Winter, Comput. Phys. Commun. 167 (2005), 195 doi:10.1016/j.cpc.2005.01.003 [arXiv:hep-ph/0407333 [hep-ph]]. 